\title{
Bilateral Renal Anastomosing Hemangiomas: A Tale of Two Kidneys
}

\author{
Hamid Abboudi, MBBS, MRCS, Benjamin Tschobotko, MBBS, \\ Christopher Carr, MBBS, and Ranan DasGupta, FRCS(Urol), MD ${ }^{1}$
}

\begin{abstract}
Background: Renal anastomosing hemangioma (RAH) is an extremely rare benign vascular tumor first described in 2009. Making this diagnosis is fraught with challenges. Radiologically they share features consistent with renal cell carcinomas (RCCs). Their vascular nature poses risks if considering preoperative biopsy and histologically they share characteristics akin to angiosarcomas. The few reports published in the literature suggest presentation with hematuria, flank pain, and polycythemia although the majority are diagnosed at postnephrectomy histologic examination. This case represents the first metachronous RAH in the literature, and is the first RAH presenting with severe hemorrhage.

Case Presentation: A 62-year-old woman of Albanian heritage presented to urology with visible hematuria and positive urine cytology. Three years before this presentation, she had undergone an elective radical right-sided nephrectomy for a suspected RCC detected on magnetic resonance imaging, which proved to be an RAH after postoperative histologic examination of the specimen. The patient was investigated with cystoscopy and ureteroscopy for this new hematuria presentation, both of which were unremarkable. Fourteen hours post ureteroscopy, the patient became severely hypotensive and developed acute kidney injury. A CT scan indicated a large left-sided renal subcapsular and retroperitoneal hematoma that was actively bleeding. The patient was hemodynamically unstable and, therefore, required an emergency open left-sided nephrectomy, rendering her anephric and dialysis dependent. Postoperative histologic examination proved that the left kidney also contained an RAH.

Conclusion: The anastomosing hemangioma is an important subtype to differentiate from angiosarcoma before and after a nephrectomy. Urologists should carefully consider invasive tests in patients with previously diagnosed vascular lesions as there may be an increased risk of bleeding. Patients with a previously diagnosed anastomosing hemangioma may require surveillance of the contralateral kidney.
\end{abstract}

Keywords: anastomosing hemangioma, hematuria, nephrectomy, ureteroscopy

\section{Introduction and Background}

$\mathbf{R}$ ENAL ANASTOMOSING HEMANGIOMA (RAH) is an extremely rare benign vascular tumor. In 2009, Epstein and Montgomery ${ }^{1}$ found histologic and clinical evidence of this unique neoplasm with many histologic similarities to angiosarcoma. Previous case reports have presented with hematuria, flank pain, and polycythemia, and most diagnoses are made postnephrectomy. ${ }^{2}$

This is an example of a bilateral case of a newly defined, exceptionally rare tumor with a novel clinical presentation. In addition, this patient was ultimately left anephric after two urologic interventions. This case, therefore, serves as a reminder of the threat posed by iatrogenesis and that we should carefully consider the possible implications of all medical management.

\section{Presentation of Case}

A 62-year-old woman of Albanian decent presented to urology with a 3-week history of visible hematuria and an equivocal urine cytology result. Three years before this

Departments of ${ }^{1}$ Urology and ${ }^{2}$ Radiology, Charing Cross Hospital, Fulham Palace Road, London, United Kingdom.

(C) Hamid Abboudi et al. 2017; Published by Mary Ann Liebert, Inc. This is an Open Access article distributed under the terms of the Creative Commons Attribution License, which permits unrestricted use, distribution, and reproduction in any medium, provided the original work is properly cited. 

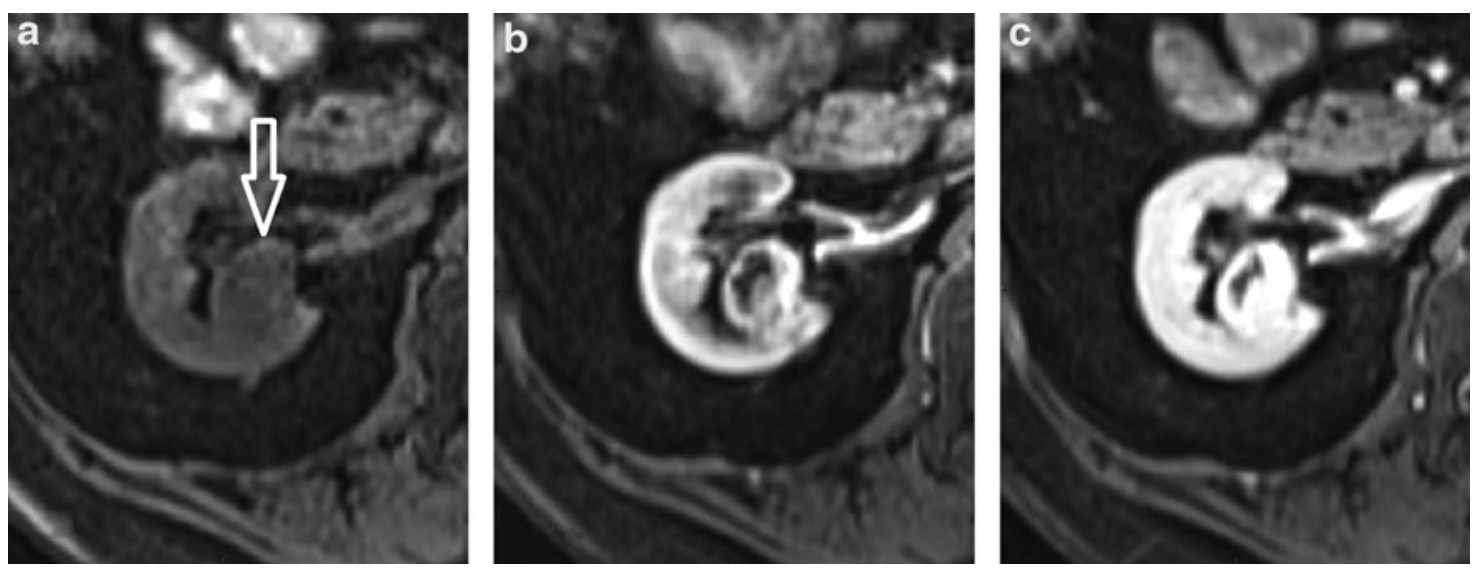

FIG. 1. Dynamic contrast-enhanced magnetic resonance images showing the $2 \mathrm{~cm}$ lesion in the interpolar region of the right kidney (arrow). In the unenhanced sequence (a) the lesion is isointense to adjacent renal tissue. In the arterial phase (b) the lesion shows peripheral enhancement and in the portal venous phase (c) there is enhancement of the central components in keeping with a solid tumor.

presentation she had undergone an elective radical rightsided nephrectomy for a suspected $22 \times 26 \times 27 \mathrm{~mm}$ renal cell carcinoma (RCC) detected on magnetic resonance imaging (MRI) (Fig. 1), which proved to be an RAH after postoperative histologic examination of the specimen. Her medical history also included chronic kidney disease (CKD) secondary to antineutrophil cytoplasmic autoantibodies vasculitis and pulmonary fibrosis. Her CKD meant she was unsuitable for a contrast-enhanced CT scan.

The patient was investigated with cystoscopy and ureteroscopy for this new hematuria presentation, both of which were unremarkable. Fourteen hours post ureteroscopy, the patient became severely hypotensive (BP 80/40) and tachycardic (heart rate 120). Her serum profile indicated severe anemia with a hemoglobin of $4 \mathrm{~g} / \mathrm{L}$, acute chronic kidney injury with a creatinine of $400 \mu \mathrm{M}$, and hyperkalemia of $\mathrm{K} 6.8 \mathrm{mM}$. A CT scan indicated a large left-sided renal subcapsular and retroperitoneal hematoma that was actively bleeding (Fig. 2).

Immediately after the identification of the actively bleeding hematoma, the patient was stabilized with transfusion of $3 \mathrm{U}$ of packed red blood cells and hemofiltration before undergoing an emergency left-sided open nephrectomy, rendering her anephric and dialysis dependent. After the left nephrectomy, the patient spent a short time on intensive therapy unit where she received renal support. She was discharged from hospital on day 7 postoperatively and is currently on the waiting list for a renal transplantation. Postoperative histologic examination proved that the left kidney also contained an RAH.

\section{Discussion and Literature Review}

The term anastomosing hemangioma was first used in 2009 by Epstein and Montgomery. ${ }^{1}$ Hemangiomas themselves are more commonly located in the skin and subcutaneous tissues. Visceral hemangiomas are generally not common and occur mostly in the liver. Epstein and Montogemery suggested that although rare, urinary tract angiosarcomas contributed a larger proportion of vascular tumors than those found in the soft tissues. They, therefore, hypothesized that some urinary tract vascular lesions may have been incorrectly diagnosed as angiosarcomas. Interestingly, they found evidence of a unique neoplasm - the RAH - a tumor that has many resemblances to angiosarcoma, with some subtle histologic differences: anastomosing hemangiomas have a backdrop of nonendothelial supporting elements, an overall lobulated configuration, and an association with medium-sized vessels, which would be unusual in angiosarcoma. In addition, they lack multilayering of endothelial cells and mitotic activity. ${ }^{1}$

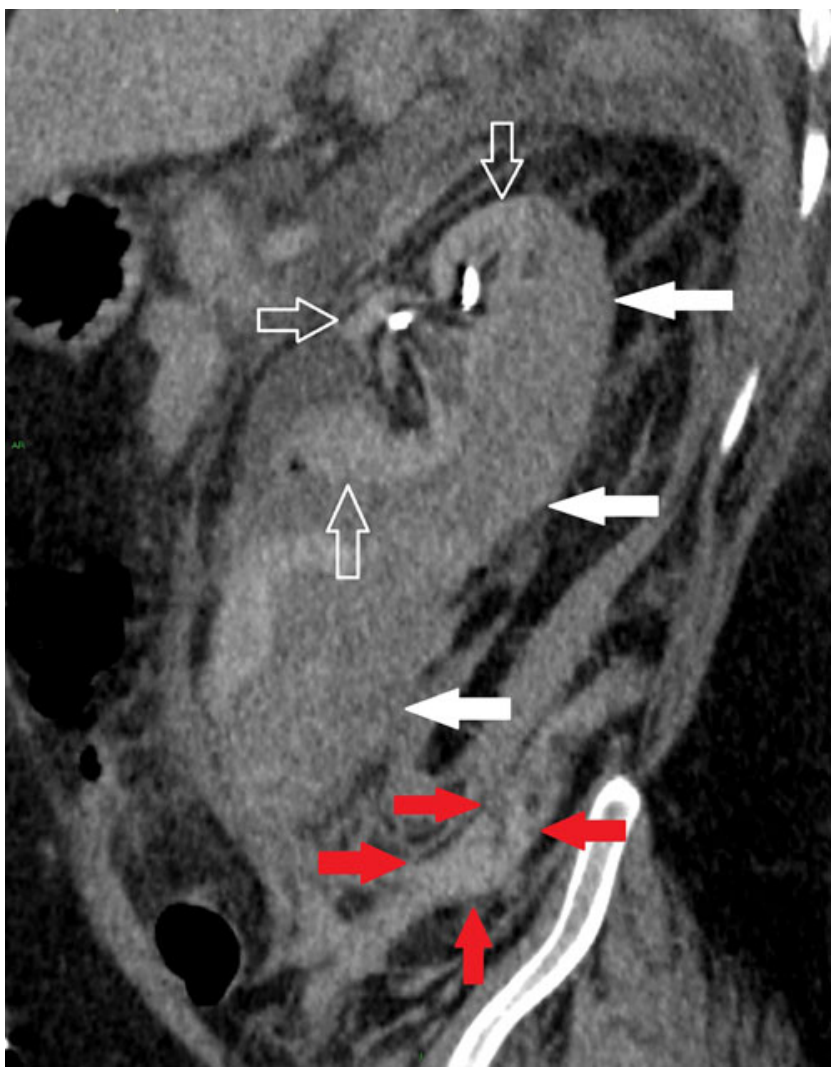

FIG. 2. Contrast-enhanced CT, oblique coronal MPR, showing the atrophied native left kidney (unfilled arrows) with a surrounding subcapsular hematoma (white arrows). There is also blood within the retroperitoneum (red arrows). $\mathrm{MPR}=$ multi-planar reconstruction . 
Importantly, the biological behavior of anastomosing hemangiomas is that of a benign lesion and they are classified as benign vascular tumors.

This case highlights the importance of differentiating the RAH from malignant lesions (both vascular and nonvascular). Unfortunately, the MRI appears to be similar to that of a malignant lesion and preoperative histologic diagnosis is difficult because biopsying vascular lesions can lead to significant bleeding. Furthermore, histologic characterization between an angiosarcoma and an RAH is challenging because of histopathologic similarities. ${ }^{1}$ In a series of eight RAHs, Kryvenko found that the anastomosing hemangioma is enhanced during the arterial phase of contrast infusion on $\mathrm{CT}$ akin to an RCC. ${ }^{3}$

In terms of management, benign vascular lesions do not necessitate radical nephrectomy and patients can immediately join the renal transplant list if required, unlike those undergoing a nephrectomy for a renal malignancy.

Normally, anastomosing hemangiomas present with hematuria, flank pain, or polycythemia. ${ }^{2}$ This is the first case wherein the patient has presented with a life-threatening hemorrhage. It, therefore, raises questions as to whether caution should be applied when contemplating invasive procedures in patients with a previous anastomosing hemangioma, even if the site of the known lesion is not being investigated. We hypothesize that the increased renal pressure from the ureteroscopic irrigation may have led to the spontaneous rupture of the RAH; fluoroscopy images demonstrate the safe passage of the ureteroscope and normal appearances within the pelvis until the subsequent (secondary) hemorrhage was detected. It is difficult to pass beyond conjecture for the cause of this bleed.

Furthermore, despite the underlying pathology analysis showing bilateral benign vascular tumor, this lady has been left anephric. The diagnosis of the anastomosing hemangiomas in this patient and most other documented cases has been made postnephrectomy. This highlights the need for improved recognition, investigation, and management of this newly discovered pathology.

The most recent and largest review of the published literature on this rare entity included 45 patients with a mean follow-up of 26 months (range 1-115 months), of which 4 had bilateral disease and there was limited evidence for disease recurrence. ${ }^{4}$

This appears to be the first case in the literature of metachronous RAH. In our unit, we have, therefore, recommended a surveillance strategy for unilateral RAH diagnoses in patients.

\section{Conclusion}

The RAH is an important subtype to differentiate from angiosarcoma before and after a nephrectomy. Urologists should carefully consider invasive tests in patients with previously diagnosed vascular lesions as there may be an increased risk of bleeding. Patients with a previously diagnosed RAH likely require surveillance of the contralateral kidney.

\section{Disclosure Statement}

No competing financial interests exist.

\section{References}

1. Montgomery E, Epstein JI. Anastomosing hemangioma of the genitourinary tract: A lesion mimicking angiosarcoma. Am J Surg Pathol 2009;33:1364-1369.

2. Zhao M, Li C, Zheng J, et al. Anastomosing hemangioma of the kidney: A case report of a rare subtype of hemangioma mimicking angiosarcoma and review of the literature. Int $\mathbf{J}$ Clin Exp Pathol 2013;6:757-765.

3. Kryvenko O, Gupta N, Meier F, et al. Anastomosing hemangioma of the genitourinary system eight cases in the kidney and ovary with immunohistochemical and ultrastructural analysis. Am J Clin Pathol 2011;136:450-457.

4. Omiyale A. Anastamosing hemangioma of the kidney: A literature review of a rare morphological variant of hemangioma. Ann Transl Med 2015;3:151.

Address correspondence to: Ranan DasGupta, FRCS(Urol), MD Department of Urology Charing Cross Hospital Fulham Palace Road London W6 8RF United Kingdom

E-mail: ranan.dasgupta@imperial.nhs.uk

$\begin{aligned} & \text { Abbreviation Used } \\ \mathrm{CKD} & =\text { chronic kidney disease } \\ \mathrm{CT} & =\text { computed tomography } \\ \mathrm{MRI} & =\text { magnetic resonance imaging } \\ \mathrm{RAH} & =\text { renal anastomosing hemangioma }\end{aligned}$

Cite this article as: Abboudi $\mathrm{H}$, Tschobotko B, Carr C, DasGupta R (2017) Bilateral renal anastomosing hemangiomas: a tale of two kidneys, Journal of Endourology Case Reports 3:1, 176-178, DOI: 10.1089/ cren.2017.0018. 